\title{
Science and the Spectrum of Belief
}

\author{
An Interview with Leonard Ornstein \\ by Peter J. Denning
}

\section{Editor's Introduction}

I recently discovered that cell biologist Leonard Ornstein had, in 1965, written a long and thoughtful essay on information and meaning. Shannon's idea that communication systems could transmit and process information without regard to its meaning just did not seem right to him. He was particularly interested in how scientists use and interpret information as part of science. He devised a simulation of a discovery process that consisted of sifting through data looking for recognizable classes and finally classifying each individual item into one of the classes. His process was obviously implementable by a computer, although the computers of the day had insufficient capacity to take on large data sets such as those found in his field. I was struck by the similarity with the modern Bayesian "autoclass" programs, which were designed much later and automated the inference process described by Ornstein. I thought it would be fascinating to talk to him and find out more about how he sees science, discovery, information, and meaning. The result is this interview.

Born in 1926, Leonard Ornstein has had a long career covering cell biology, cytochemistry (first high-resolution methods for esterases and phosphatases), flow-cytometry, electrophoresis, bioengineering, biophysics, electro-optics, optical and electron microscopy (first images of internal structure of mitochondria, cilia and flagella, pores of nuclear membranes, epithelial brush borders, myoneural junctions, electroplax), unsupervised learning, information theory and meaning, pattern recognition and artificial neural networks, automated medical diagnosis, epistemology, agricultural irrigation, and global warming. He received his Ph.D. from Columbia University in 1957 and taught and performed research in their Zoology Department from 1949 until 1964. He joined Mt Sinai Hospital in 1954 and, in 1966, became a professor of pathology at Mt. Sinai School of Medicine. He retired from Mt. Sinai in 1992 and has remained an emeritus professor to the present. He has consulted for numerous companies on medical technology and instrumentation and has been a principal in three businesses (two terminated in 2003, and the last was sold in 2006). He is best known for pioneering a technique called polyacrylamide gel electrophoresis, for the analysis of proteins and nucleic acids, in the 1950s. He has been married for 68 years and has four children, nine grandchildren, and six great grandchildren.

Peter J. Denning Editor-in-Chief 


\section{Science and the Spectrum of Belief \\ An Interview with Leonard Ornstein \\ by Peter J. Denning}

Ubiquity: We are here to discuss what you see as deterioration of public discourse about the role of science in important problems we face in our society. You have been involved in science for a long time and have a unique perspective on this issue. Let's begin with a summary of your own interest in science.

Leonard Ornstein: I've been interested in science all my life. While much of my work was in cell biology, I have had a long interest in information - not only in the way that cells carry information but in the way that scientists learn information from the "signals" visible in our environments. This brought me early on to Shannon's information theory, which intersects strongly with computer science. In more recent years I have been interested in how information moves in public discourse.

Since 2006, I have worked on a method to rescue the Sahara desert from its eternal drought and turn it into a productive irrigated forest that could end global warming. This would more than counteract the destruction of tropical forests, which is one of the two big drivers of global warming. To address the second driver-burning of fossil fuels-I have also worked on the most effective ways to manage tropical forests so that they can sustainably provide sufficient wood as a permanent replacement for fossil fuels, without adding to global warming. In my engagement with the public aspects of these scientific issues, I have been appalled at the poor quality of the discourse. Many claims and counterclaims are based on rather weak evidence. However, global warming remains very serious even if the mean warming rates turn out to be only half the $0.2^{\circ} \mathrm{C}$ per decade plausibly projected by most climatology experts.

The way my own scientific work has been cited-or not-illustrates inefficient use of the scientific record. Over the years, my 135 publications and patents have been cited more than

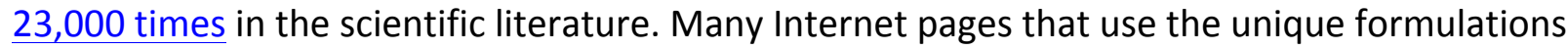
and inventions of disc electrophoresis did not cite the original work by my late long-term partner B. J. Davis and me. Through appropriate Google searches I learned there are about 8 million such pages exhibiting this use.

[Analysis available at http://www.pipeline.com/ /enornst/DiscElectrophoresis.html]. 
People who read these pages have about one chance in 350 ( 8 million divided by 23,000 ) of coming across a citation to the work being used. The people who write these pages are providing results of methods, without also providing access to the science behind the methods. How can someone reading their papers learn their full significance without a path back to the source? This is terribly sloppy practice. I believe that such dilution of citation of methodological background tends to seriously degrade and slow progress in general education, in science education, in invention, and in innovation.

Ubiquity: What are some of the areas in society, business, and commerce where science can provide significant improvements?

LO: Science is heavily involved in most of the big controversies of our day. Some examples are

- The role of human activities that cause global warming, an issue known as anthropogenic global warming (AGW).

- The role of natural selection in evolution.

- Failing educational systems from grade schools to universities.

- Security and privacy of information.

- Incompatibilities between "free speech," "free markets." and "truth in advertising."

- The paradox of simultaneously increasing productivity, joblessness, and income inequality.

Let me comment on the last of these items. The accelerating efficiencies of automation, of wide-band communication and of other fruits of science are virtually ignored as the basic problem in joblessness. Politicians focus on gross domestic product (GDP), and growth in capital, rather than quality of life (QOL), as appropriate prime economic motivators. They invoke discredited economic ideologies (e.g., Say's Law or its Keynesian substitutes) instead of well-supported scientific models.

Most professional economists-academic, corporate, media and governmental- are ideologues, not scientists. They do not understand, and are not willing, as science requires, to challenge their models with relevant observations of the way markets work. For example, most libertarians, free-market supporters in principle, object to market regulation. They focus on its negative effect on GDP, rather on faulty design of particular implementations of regulations. They seem unwilling to recognize that well designed regulation levels the playing field and equalizes opportunity, leading to more-rather than less - freedom, and to increase in QOL. An 
example is good rules-of-the road: Regulations designed to maximize the flow and safety of traffic for the QOL of both pedestrians and motorists.

When production per man-hour goes up, fewer employees produce more goods and services. But the growing cadre of unemployed cannot afford to consume, and demand for nonessentials goes down. There are fewer people to consume what is being produced. It would appear that Malthus's model of the necessary exhaustion of non-renewable resources as populations grow exponentially is finally leading to technological unemployment and a market that cannot be repaired with either increased demand or supply.

Before we leave this question, let me give a contrasting example of a hard problem being approached through ideological (non-scientific) arguments. This is the impasse, in the U.S. House of Representatives and Senate, on the conflicting problems of how to both distribute the burden of public debt and also increase quality of life (QOL). Both sides invoke phrases from the Constitution to support their conflicting proposals - "insure domestic tranquility," "provide for the common defense," "promote the general welfare and secure the blessings of liberty to ourselves and our posterity," and "establish justice." Ideology shows up because the conflicts arises from differing underlying beliefs that the parties are unaware of, and are not willing to open themselves to questioning of those beliefs.

Ubiquity: You mentioned that regulation could be helpful. Some people worry that it is too easy to overdo regulation and wind up with less of what the regulation is supposed to promote.

LO: The way I see it is that various "freedoms" maximize opportunities for the introduction and expression of new ideas (models) in order to provide equality of opportunity to increase QOL. But there is a problem, that people with special advantages such as control of special resources or insider connections can take over the system. The purpose of well-designed regulation is to prevent those with special advantages from monopolizing control. One of the tools used by those seeking control is abuse of the privilege of free speech to spread exaggerated and false claims. Examples are the claims by proponents that "conventional," "over-the-counter," and "alternative" medical procedures are safe and effective based on "successful clinical trials." More often than not, the clinical trials cited fail to live up to the agreed-upon, required, scientific standards of measurable levels of confidence in the claimed observational tests of effectiveness. "Free" and "regulated" must go together. We are so inundated in advertising that deception-intentional or innocent-can have a profoundly negative effect on QOL. But regulations must be crafted very carefully to try to police only such anti-social excesses of otherwise unrestricted freedom. What libertarians call "freedom within the law" attempts to 
capture this idea, but the rhetoric of most libertarians and conservatives sounds as though they abhor all regulations.

Ubiquity: Have policy makers and thought leaders fallen into traps that prevent them from making progress with these issues?

LO: Yes. One, as noted above, is focusing on gross measures such as GDP and ignoring measures of individual QOL. The other is discussing various economic, religious, and scientific "truths" as if they can be shown to be absolute and free of uncertainties.

Ubiquity: The evolution debate is a good example of clashes between religious truths and scientific truths. What do you make of this?

LO: They are in different domains. When we talk about how scientific findings can help solve a problem, we need a much better understanding of "truthfulness." In science we deal with inductive claims and deductive claims about models of reality. Inductive claims are generalizations made from patterns observed in limited sets of data. It is easy to see that we cannot be certain about generalizations because we are finite beings whose views and descriptions of the big picture are necessarily incomplete. Deductive claims look safer, but they are not. These claims are derived, without error, from a set of agreed upon axiomatic rules, definitions, and associated meanings. I am particularly concerned to clarify the uncertainty associated with deductive claims. Even when the logic is impeccable, these claims can never be flawless because they always depend on agreed upon axiomatic elements that are recognized to be arbitrary and unprovable. This is good reason to be uncomfortable about some consequences of logical deductions.

We use verbal and other symbolic communications to share our private and group experiences of the world. With a little introspection, it is not hard to see that descriptions of "experiences" are stripped down models of sets of mental images (particularly visual) of perceptions and interpretations that include many details for which we have no words. We couch these models as stories, speculations, hypotheses, theories, laws, and the like. We wind up trying to communicate complex phenomena with very simple, stripped down sketches. Thus, the quality of deductions from those models is always open to question. 
Ubiquity: Making new hypotheses is a central practice of science. How do we measure uncertainty of our hypotheses?

LO: Let's clear up one misunderstanding. In science, the main role of induction is not generating hypotheses, but increasing (or decreasing) certainty for proposed models and hypotheses. In new models especially, there is always uncertainty about whether the model applies in new cases that have not been seen or tested before. Over time, as evidence accumulates in favor of the hypothesis, the more believable it becomes. It is important to note that uncertainty is social. It does not matter how sincerely I believe in my hypothesis-others may be unconvinced. Uncertainty in the community will diminish as sufficient testing produces increasingly convincing evidence. When enough people accept a hypothesis as true, it will tend to be accepted as a "fact" by much of the community and is likely to be treated as unqualified "truth" in future discourse. This usually goes too far!

Ubiquity: So some of the beliefs I cherish as true might have been uncertain hypotheses at some earlier time?

LO: Yes. Axioms are actually the foundations of all beliefs. Understanding the world in terms of plane geometry is a good example. Plane geometry demands adherence to Euclid's famous axioms: (1) a straight line can be drawn joining any two points; (2) any straight line segment can be extended indefinitely in a straight line; (3) given any straight line segment, a circle can be drawn having the segment as a radius and one end point as center; (4) all right angles are congruent; and (5) if two lines are drawn which intersect a third in such a way that the sum of the inner angles on one side is less than two right angles, then the two lines inevitably must intersect each other on that side if extended far enough (the "parallel postulate"). Many people believe the world adheres to plane geometry without being aware of Euclid's axioms.

But there is more. In accepting plane geometry, we agree (or think we do) on the axiomatic intended meanings of words such as "straight," "line," "plane," "point," "circle," "radius," "extended," and "indefinitely." Unfortunately, this is a bit more than the little most people know (or care to know) about axioms. As you know, there are many disagreements that would never have arisen had the participants realized they were using the same words but with different intended meanings.

In non-scientific domains or ideologies, one must believe the axioms or one will not be accepted as a member of the community. This is why it can be hard to question beliefs-one can be exorcised from the community for even suggesting any serious discussion of uncertainties about its axioms. 
Ubiquity: Have scientists lost sight of these sources of uncertainty?

LO: I think so. Many scientists make claims that appear to them to be true because they are deductively logical, but they do not understand that their own beliefs (and everyone's' beliefs of all kinds), on which the logic is based, must depend on unprovable arbitrary axiomatic agreement on rules, definitions, names, labels, and codes. Most teachers in schools do not understand this basic aspect of truth, and their students show it. I would like to help people understand these basics of logic and science so that they may conduct themselves "better," and with understanding of beliefs in general, but especially in public and private debates.

A major message of science, not shared by ideologies, is that the value of scientific models depends on the amount of relevant observational evidence brought forward in support, and on the willingness of others to accept the evidence and the models-and indeed to base that acceptance on mutually agreed upon measures of confidence. In science, it does not matter how carefully we reason about and deduce properties of stories, models, hypotheses, theories, predictions, and projections. Unless these statements are confidently supported by pertinent observations and by other empirically well-supported science, they are often-but not alwaysworthless.

Ubiquity: You have referred to a Spectrum of Belief. What is that?

LO: It is a way of visualizing the different levels of grounding (mutually agreed upon axiomatic foundations) behind beliefs. The grounding is the arbitrary commitment that supports the belief. I invite interested readers to look at my essay on the topic "The Skeptical Scientific MindSet in the Spectrum of Belief."

Ubiquity: The word "spectrum" suggests a continuum with two ends. Can you give examples of belief systems and their positions in the spectrum? For example, where do economic ideological beliefs, which you mentioned before, fall? Or pseudo-science claims?

LO: At one end of the sprectrum are ideologies that allow no challenges to their axioms and logically-derived conclusions. At the other end are scientific beliefs that are axiomatically required to subject all their models to relevant empirical challenge. There are gradations within these categories depending on the amount of grounding the system accepts. For example, an ideological axiom like Say's law, whose proponents are not allowed to question, is at the far 
ideological end. A Malthusian claim backed up with evidence of resource depletion is more toward the middle but may still have insisted that exponential depletion is certain even when the evidence was not yet there. A pseudo-scientific claim such as planet conjunction in astrology might be in the middle because it advance some evidence while still insisting on some of its beliefs even if unsupported by direct evidence. Normal science is toward the other end, and parts of science with high certainty based on extensive evidence are at the extreme end.

New models present difficulties. String theory in physics is a carefully crafted model that has not be subjected to experimental test, and may ultimately be untestable. It is difficult to distinguish from pseudo-science. Pseudo-science involves models that consistently fail empirical testing and appear incompatible with well-confirmed science; they are ideological. String theory may or may not one day become a true part of science.

I believe there is a large pool of people who do not make these distinctions and accept ideological or pseudo-science models as readily as true science. They apply inconsistent "logic" and ignore errors and uncertainties and their beliefs are mainly worthless nonsense. This gets to be a real concern when they have an influence on policy decisions.

Ubiquity: Does science occupy a special place in the spectrum?

LO: Yes. No other system of discourse matches science in its ability to provide useful, ways to understand the world and improve quality of life (survival, convenience and comfort).

Ubiquity: You just stated a belief about science. How well grounded is it?

LO: My belief is based on three claims. First, as I noted earlier about my essay, our names and stories about experiences are shallow caricatures of a rich and complex set of conscious and subconscious mental images and impressions. The language we use to communicate experiences is sparse compared to the experiences themselves. Therefore, it is extremely unlikely that any models express the absolute (and final?) truth about worldly realities.

Second, applied sciences are responsible, directly or indirectly, for virtually all the recognized increases in quality of life of the last few centuries. They are constantly subject to revision that might increase utility and are also subject to further relevant observational testing that can revise levels of confidence in their models. 
Third, scientific models of phenomena observable in the world, and ourselves, provide simpler and more reliable "explanations" than those of non-scientific disciplines and ideologies. Ockham's Razor - the dictum to choose the simplest explanation, all other things being equalgenerally places scientific models ahead of ideologic models of observable phenomena. I prefer that policy makers would use grounded scientific models rather than those of their own ideologies to make real-world decisions.

Ubiquity: You have outlined a model of science itself, in which scientists make claims that are evaluated by their community, and only those claims that become convincing to enough people through relevant supporting evidence approach certainty. In his famous book, The Structure of Scientific Revolutions, Thomas Kuhn offered a different model based on belief systems being occasionally disrupted by anomalies. Can you outline Kuhn's model and compare it with yours?

LO: Kuhn stated, that before 1962, scientific progress had been seen primarily as "development-by-accumulation" of accepted facts and theories. He called this "normal science." He argued for an episodic model in which periods of normal science are interrupted by periods of revolutionary science. Kuhn argued that an accumulation of anomalies that cannot be explained by the current belief system of science forces a switch to a new belief system. The switch may be perceived as a revolution. He used the term "paradigm" for a scientific belief system, and "paradigm shift" for the revolution.

As an example of a switch, Kuhn cites the transition from the Ptolmeyan model of the solar system, where planets followed complicated epicyclic orbits around the earth, to the Copernican model, where planetary bodies follow conic-section orbits around the sun-as a "paradigm revolution." Well, that's not how I see it. The switch was merely the slowly gained appreciation that (in the Ockham's Razor sense), the Copernican model was much simpler. It gave simpler and more accurate calculations of planetary positions. It gave everyone a clearer appreciation of what was seen in the sky.

Although Kuhn talked about belief systems, he did not discuss how we come to have our beliefs or what axioms they are founded on. In my work I have come to appreciate a difference between a belief statement as a set of words and the intended meaning of the statement. $A$ scientific belief is intended to convey meaning, not just transfer some information represented as symbols.

My own personal experience of moving between scientific models does not conform to Kuhn's idea. I did not experience a wrenching shift of beliefs to remove anomalies; I experienced the 
satisfaction of finding a simpler model that explained all the observations. (See "Tenuous but Contingent Connections."

Ubiquity: Now you are sounding like information theory. What is the relation of your work with information theory?

LO: In their famous 1949 paper "The Mathematical Theory of Communication," Claude Shannon and Warren Weaver state, "the semantic aspects of communication are irrelevant to the communication aspects." I was interested in this because it seems like scientific discovery could be modeled as a communication problem, from nature to human, and yet humans are deeply interested in the meanings of what they discover. In 1965 I wrote a paper, "Computer Learning and the Scientific Method: A proposed solution to the Information Theoretical Problem of Meaning" that looked deeper into the meaning (semantic) question. There I simulated a model of a classification system that automatically discovers classes among large numbers of input patterns, generates operational definitions of class membership with explicit levels of confidence, creates a continuously-updated, "self-organized," coded, hierarchical, taxonomic classification of patterns, and recognizes to which already discovered class or classes, if any, a new input belongs.

I would add, as an aside, that this strategy is similar to the one used in some Bayesian inference systems. However, Bayesian methods of classification and learning depend on an unprovable axiomatic commitment: a "Bayesian Prior," which is a "guess" at the prior probability distribution of the data being studied. All Bayesian systems depend on such guesses. Beside mine, I can think of two other examples of classification and learning methods that do not require such a guess: The artificial intelligence methods that now go under the name of "learning without a teacher," and frequentist statistics. A preference for Bayesian or nonBayesian methods is a choice among beliefs-and, as I have been saying, a choice as to which axioms to believe cannot be defended with logic.

I demonstrated that Shannon and Weaver were wrong about the possible relevance of meaning (semantics) to communication aspects of information theory. The naming of the classes of the automatically-generated information tree are not arbitrary. They convey meaning to human observers. When used as the codes for the branches of a classification tree, meanings are less easily disrupted by noise than the arbitrary codes of information theory. I took another look in 1969, in "Hierarchic Heuristics: Their relevance to economic pattern-recognition and high-speed data processing." I showed that hierarchical, organizations-business, academic, labor-union, and governmental-also permit potential efficient transfer of intended meaning up and down 
the bureaucratic tree for the very same reasons. Hierarchic trees have very high efficiency that varies less than a factor of 1.5 from 2 to 10 branches per branch point. However, when meaning is corrupted in such transfers along the tree, by intention or incompetence, the advantages are lost. I conclude that meaning-transfer corruption is the real cause of common dissatisfaction with many bureaucracies, not the usual suspects of "bloat" and "inefficiency."

Ubiquity: I agree that the switch from Ptolemy to Copernicus can be seen as a shift to a simpler, more accurate model. But what about Einstein and relativity? Einstein said he struggled with a paradox of 19th century physics, namely the conflicting claims that all motion is relative to the observer (Lorentz), and that the speed of light is the same for all observers (Michelson and Morley)? Einstein created a new theory in which both old claims were simultaneously true. How can that be seen as a switch to a simpler model?

LO: Relativity is simpler in the sense that it permits description of observable events in a less complex and more consistent manner than Newtonian, Maxwellian and Lorentzian models. However, I would agree that Einstein's Relativity Theories are excellent candidates for Kuhn's "paradigm shift" model.

Ubiquity: Are there other belief systems about science?

LO: Daniel Dennett-in his book The Intentional Stance-says, "Here is how it works: first you decide to treat the object whose behavior is to be predicted as a rational agent; then you figure out what beliefs that agent ought to have, given its place in the world and its purpose. Then you figure out what desires it ought to have, on the same considerations, and finally you predict that this rational agent will act to further its goals in the light of its beliefs. A little practical reasoning from the chosen set of beliefs and desires will in most instances yield a decision about what the agent ought to do; that is what you predict the agent will do." Dennett's description is widely acclaimed as a modern description of how science dispenses with religious and ideological beliefs. One might suppose that his focus on "intentions" would tie in with axiomatic "intended meaning." But he virtually completely ignores discussion of the role of axioms and the roots of what he calls "a rational agent." How can you know what an agent believes without knowing the agent's axioms? His model is very different from mine.

Richard Dawkins, in his 1976 book The Selfish Gene, coined the term "selfish gene" as a way of expressing the gene-centered view, as distinct from organism-centered or the group-centered view. In the gene-centered view, the individual's genes drive action, not the individual's social 
group. This model is also widely cited as a way for science to dispense with the need for religions and ideologies. But there is an obvious problem: From the gene-centered view, the more two individuals are genetically related, the more sense (at the level of the genes) it makes for them to behave selflessly with each other. Try to explain family feuds with that! Dawkins's explanation of family feuds seems as unsatisfactory as the Ptolmeyan epicyclic model of the solar system. Chimpanzees, with whom we share about 99 percent of our genes, are not normally treated as kin. But somehow, the unshared 1 percent of their genes overwhelms the selfishness of the 99 percent, producing altruism towards humans! This does not fit the Selfish Gene model very well.

Although both Dennett and Dawkins share my prejudice that survival, convenience, and comfort must originally have "motivated" the evolutionary origin of communication of shared intended meanings, they do not follow up by analyzing just what such communication-and reasoning-entails.

Ubiquity: Where do you place the Kuhn, Dennett, and Dawkins belief systems in your Spectrum of Beliefs?

LO: As scientific, rather than ideologic models-but with marginal empirical support.

Ubiquity: Do you have some recommendations on how we can get your model into play? How would it help advance the discourse on the tough issues you listed at the start of this conversation?

LO: When you work consistently within a discipline and earn respect for your performance, the members of that discipline watch for, and generally will read your latest writings. However, when you publish outside your main discipline, your reputation in science may not carry much weight. It matters that you can generate a following for your ideas. That is tough. I have tried sending copies of my writings to others whose recent publications and public reports indicate that they should have a high interest in subjects closely related to my models. And I also post comments on threads on blogs that relate closely to my models. But this has not proved to be a reliable way to generate a larger following. So I do not really have a good answer to your question.

The central idea I have been working with - the grounding of claims and beliefs-is much easier to put into practice than other science models such as Kuhn's, Dennett's, or Dawkins'. It is not obvious how to change a paradigm, but it is obvious that I can try to show that my own claims 
are well grounded and that it is possible and desirable to understand foundational beliefs. By practicing and teaching that, I may contribute to better public discourse and to better science.

\begin{abstract}
About the Author
Peter J. Denning is the editor-in-chief of Ubiquity and a past president of ACM (1980-82). Currently he is a distinguished professor, chair of the Computer Science Department, and director of the Cebrowski Institute at the Naval Postgraduate School in Monterey, CA.
\end{abstract}

DOI: $10.1145 / 2447476.2447477$ 